\title{
Randomised trial of block and replace vs dose titration thionamide in young people with thyrotoxicosis
}

\author{
Claire L Wood 1,2, Michael Cole 3 , Malcolm Donaldson ${ }^{4}$, David B Dunger ${ }^{5,6}$, Ruth Wood ${ }^{7}$, Niamh Morrison ${ }^{1}$, \\ John N S Matthews ${ }^{3,8}$, Simon H S Pearce ${ }^{2,9}$ and Timothy D Cheetham ${ }^{1,2}$ on behalf of the British Society for \\ Paediatric Endocrinology and Diabetes (BSPED)
}

${ }^{1}$ Department of Paediatric Endocrinology, Royal Victoria Infirmary, Newcastle-upon-Tyne, UK, ${ }^{2}$ Translational and Clinical Research Institute, Faculty of Medical Sciences, Newcastle University, International Centre for Life, Central Parkway, Newcastle upon Tyne, UK, ${ }^{3}$ Population Health Sciences Institute, Newcastle University, Baddiley-Clark Building, Newcastle upon Tyne, UK, ${ }^{4}$ Department of Child Health, University of Glasgow School of Medicine, Glasgow, UK, ${ }^{5}$ Department of Paediatrics, University of Cambridge, Cambridge Biomedical Campus, Cambridge, UK, ${ }^{6}$ Wellcome Trust-MRC Institute of Metabolic Sciences, University of Cambridge, Cambridge, UK, ${ }^{7}$ Newcastle Clinical Trials Unit, ${ }^{8}$ School of Mathematics, Statistics \& Physics, Newcastle University, Newcastle-upon-Tyne, UK, and ${ }^{9}$ Department of Endocrinology, Royal Victoria Infirmary, Newcastle-upon-Tyne, UK

Correspondence should be addressed to T D Cheetham

Email

tim.cheetham@nhs.net

\begin{abstract}
Objective: First-line treatment of thyrotoxicosis in young people is thionamide anti-thyroid drug (ATD) in a blocking dose with levothyroxine replacement (block and replace, BR) or in a smaller dose tailored to render the patient euthyroid (dose titration, DT). Our objective was to determine which regimen provides more stable biochemical control.

Design: A multi-centre phase III, open-label randomised trial comparing BR with DT in patients aged 2-17 years with newly diagnosed thyrotoxicosis at 15 UK centres.

Methods: Patients were randomised shortly after diagnosis and treated for 3 years. The primary outcome was the percentage of serum thyroid-stimulating hormone (TSH) levels in the reference range between 6 months and 3 years. Secondary outcomes included the proportion of Free thyroxine (FT4) levels in the reference range, adverse event frequency and 4 years outcome (remission/relapse).

Results: Eighty-two patients were randomised, with details on clinical course in 81 (62 Female); 40 were allocated to BR (41 DT). Three withdrew with one ineligible. The mean percentage of serum TSH within reference range was $60.2 \%$ in BR and $63.8 \%$ in DT patients; adjusted difference $4.3 \%, 95 \% \mathrm{Cl}(-7.8$ to 16.4$) ; P=0.48$. Proportions for FT4 were $79.2 \%$ in BR and $85.7 \%$ in DT patients; adjusted difference $6.8 \%,(-0.2$ to 15.6$) ; P=0.13$. Three patients developed neutropenia - all on BR. 6 BR and 10 DT patients were in remission at 4y.

Conclusion: This randomised trial has shown no evidence to suggest that $B R$, when managing the young patient with thyrotoxicosis, is associated with improved biochemical stability when compared to DT.
\end{abstract}

\section{Introduction}

Thyrotoxicosis in young people is usually due to Graves' disease (GD) where pathogenic antibodies stimulate the thyroid-stimulating hormone receptor (TSHR), resulting in thyroid hormone excess. The first-line treatment for most young people with hyperthyroid GD is the antithyroid drug (ATD) carbimazole (CBZ) or methimazole https://eje.bioscientifica.com https://doi.org/10.1530/EJE10.1530/EJE Printed in Great Britain

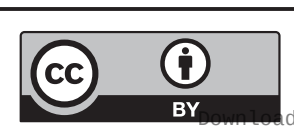

This work is licensed under a Creative Commons Attribution 4.0 International License. 
(MMZ). Propylthiouracil (PTU) should be used only in exceptional circumstances in the young because of the increased risk of severe liver damage when compared to CBZ and MMZ (1). ATD is usually administered in one of two ways. The first approach is to use a daily ATD dose that prevents endogenous thyroid hormone production completely. Thyroid hormone is then added in a replacement dose as serum thyroxine concentrations fall. This strategy is called block and replace or BR. The second approach is to commence an ATD dose that will render the individual euthyroid in the first weeks from diagnosis. The ATD dose is then progressively reduced as the thyroid hormone concentrations normalise and the patient is then maintained on an ATD dose that keeps them biochemically euthyroid. This strategy is referred to as dose titration or DT (2). Biochemical control is an important issue in childhood thyrotoxicosis because even subclinical hyperthyroidism can affect attention span, development and school performance (3). The short and long-term impact of more severe thyroid dysfunction can be profound (4). Excess thyroid hormone can result in rapid growth and osteopenia (5) whilst patients who are hypothyroid whilst on either regimen are at risk of slow growth and impaired quality of life. A recent study has demonstrated that prompt control of biochemical hyperthyroidism is associated with a better long-term vascular outcome than having persistently abnormal thyroid function $(4,6)$ which may be pertinent to the health of adolescents as they transition through into adult life.

There has been no randomised trial comparing the two approaches (DT and BR) in the young person (or indeed adults) to date. This is a concern for many clinicians because the changing thyroid requirement during rapid growth could potentially make enhanced biochemical stability particularly desirable at this time. This is also an important question because young patients may remain on ATD for a lengthy period of time $(7,8)$ and because they could be more susceptible to the side effects of ATD than adults (9). Understanding the potential risks and benefits of the two key ATD treatment strategies (BR and DT) is therefore important if young people and their families are to make appropriately informed decisions about their management.

With this background, we undertook a randomised trial of DT vs BR in young people with thyrotoxicosis to determine which regimen provided more stable biochemical control when the interval between clinic visits was the same in the two trial arms.

\section{Subjects and methods}

\section{Study design}

The trial was a multi-centre phase III, un-blinded randomised trial comparing block and replace (BR) with dose titration (DT) anti-thyroid drug treatment. The study was planned by members of the British Society for Paediatric Endocrinology and Diabetes (BSPED) and was initially managed by the Paediatric Clinical Trials Unit in Cambridge and subsequently by Newcastle Clinical Trials Unit. The trial was registered as EudraCT Number: 2011-001238-40 (DDX ref: MF8000/13328) and as NCT01436994 on Clinicaltrials.gov. A favourable ethical committee opinion was received in 2004 (Berkshire Research Ethics Committee). 15 UK paediatric units recruited patients to the trial. These units were Aberdeen, Birmingham, Cambridge, Cardiff, Coventry, Dundee, Edinburgh, Glasgow, Kilmarnock, Liverpool, London (St. George's), Manchester, Newcastle, Norwich, Sheffield.

\section{Participants}

UK-based patients with thyrotoxicosis between the ages of 2.0 and $<17.0$ years of age were recruited between January 2004 and November 2011. Patients assented or consented to take part in the trial with parental consent in those individuals under 16 years of age. Patients were diagnosed on the basis of a suppressed serum TSH (low levels that were below the assay threshold according to the local reference range) and raised thyroid hormone concentrations (above the local reference range) as well as a typical clinical picture: tachycardia, palpitations, hyperphagia, frequent stools, altered mood and weight loss. Patients with toxic thyroid nodules, McCune Albright syndrome or previous episodes of thyrotoxicosis were excluded. Patients were recruited by tertiary trained paediatric endocrinologists (https://www.eurospe.org/education/education-trainingsyllabus/) who diagnosed thyrotoxicosis on the basis of the clinical and biochemical picture. Thyrotropin receptor antibody (TRAb) titres were not routinely measured in all centres when the trial started. One patient was randomised in the first months of the trial but no follow-up data were collected at this site. We report the clinical course and outcome of the remaining 81 patients.

\section{Randomisation}

Patients were allocated to the BR and DT treatment groups in the ratio 1:1. Randomisation was conducted 
by the Paediatric Clinical Trials Unit in Cambridge using minimisation with four stratification factors which were age $(<10$ or $>10$ years), Free Thyroxine (FT4) level at presentation $(<50 \mathrm{pmol} / \mathrm{L}$ or $>50 \mathrm{pmol} / \mathrm{L})$, sex (male or female) and region (Anglia, Midlands, North-East, NorthWest, South East, Scotland Wales, Yorkshire). The allocation was done using the MINIM programme which allocated to the group minimising imbalance with probability 0.7 (10).

\section{Procedures}

Anti-thyroid drug regimen: Block and Replace regimen (BR) or dose titration (DT)

Patients were treated with ATD from diagnosis $(0.75 \mathrm{mg} /$ $\mathrm{kg} /$ day of carbimazole) and subsequently randomised to either BR or DT (details of the BR and DT study regimens are presented in the Appendix, please see section on Supplementary materials at the end of the article). This ATD dose was expected to abolish endogenous thyroid hormone production in the majority of patients. Patients in the BR arm received thyroid hormone in a replacement dose as they became eu- or hypothyroid whilst patients randomised to DT underwent ATD dose reduction as they became euthyroid. Patients were managed by paediatric endocrinologists skilled in the area of thyroid hormone replacement in childhood but an initial dose of $75 \mu \mathrm{g} / \mathrm{m}^{2}$ of thyroxine was suggested in the BR guideline.

Patients were seen regularly for the first 6 months (every 4 weeks up to 16 weeks) and then at 3 monthly intervals from week 26 until the end of year 3 . The protocol, therefore, fixed the time interval between clinic visits in the two study arms although additional, unscheduled visits could take place between these assessments as clinically indicated. Patients then stopped ATD at the end of year 3 and were followed up until the end of year 4 .

\section{ATD - Carbimazole and Propylthiouracil}

Participants received the ATD for 3 years, followed by a period of ATD therapy to determine remission or need for ongoing treatment or consideration of alternative management. Paediatricians in the UK usually commence children with thyrotoxicosis on carbimazole rather than PTU. When patients were treated with PTU a similar guideline was followed with the recommendation that $1 \mathrm{mg}$ of carbimazole is approximately equivalent to 10 mg of PTU. Clinicians adhered to local policy or their usual practice in terms of blood count and liver function monitoring.

\section{Outcomes}

Relapse and remission definition

Relapse was defined as patients with the combination of a suppressed TSH $(<0.05 \mathrm{mU} / \mathrm{L})$ and raised thyroid hormone level according to the local reference range up to the end of year 4 (4 years post-presentation, 1 year following ATD cessation).

Patients were deemed to be in remission if they did not fit the criteria for relapse at 4 years post-diagnosis, having been off ATD for 12 months and not receiving definitive treatment. The patient was kept under review by the local investigator after stopping ATD so that their status after 12 months off ATD could be documented.

\section{Statistical analysis}

Primary outcome and power calculation

The primary outcome of the study was the proportion of TSH measurements from each patient that were within the normal range according to local laboratory reference values. This variable was based on all measurements obtained between 6 months and 3 years, including unscheduled visits and was calculated centrally following data collection and submission. A clinically important difference between the treatment groups in the mean of this variable was taken to be 0.1. In other words, a difference of $10 \%$ in the proportion of TSH concentrations in the reference range when the groups were compared was deemed to be clinically important. The standard deviation of this variable was approximately 0.2 , according to preliminary data collected from patients with thyrotoxicosis managed in Newcastle upon Tyne and Glasgow. To obtain 80\% power at the two-sided 5\% level required each group to be of size 64 . The trial closed short of the required sample size, due to initial slow recruitment and then a subsequent funding shortfall.

A further analysis was conducted that excluded measurements obtained from patients after they had had definitive treatment with surgery or radioiodine (where applicable). In addition, a sensitivity analysis of the primary outcome variable was performed which excluded TSH measurements that were taken more than 4 weeks from the protocol stipulated visit date.

\section{Secondary outcomes}

Four secondary outcomes are reported: 
1. The proportion of FT4 concentrations within the local laboratory reference range, collected over the same period used for the primary outcome.

2. The remission status of the patient at the end of the study period (i.e. 4 years after recruitment).

3. The number and type of adverse events reported by each patient.

4. Four additional measures of biochemical control were computed for each patient, namely the mean and standard deviation of the TSH levels and of the FT4 levels.

The difference between the treatment groups in the proportion of TSH and FT4 within the normal range was estimated using linear mixed-effects models, with 95\% CIs being given for the adjusted mean treatment difference, and $P$-values quoted for the two-sided test using a null hypothesis of no adjusted mean difference. Adjustment was made for the stratification variables: age, sex, initial FT4 hormone concentration and region; the first three of these variables were entered as fixed binary covariates, with the region being treated as a random covariate. Estimation is by restricted maximum likelihood and was conducted using Stata Version 15 (11).

\section{Treatment following relapse}

The outcome for patients who relapsed following a course of ATD were categorised into those who subsequently returned to ATD therapy (either BR or DT) or who were treated with one of the more definitive second-line options that are associated with the ablation or removal of thyroid gland tissue (radioiodine or surgery).

Safety and adverse events were assessed according to defined criteria and all reported serious adverse events were verified against treatment notes/medical records (source data verification). Regular monitoring visits were also carried out by the Newcastle Clinical Trials Unit. The trial was overseen by a data and ethics monitoring committee. The members comprised an independent paediatrician, expert endocrinologist and statistician.

\section{Role of the funding source}

The funders had no role in study design, data collection, data analysis, data interpretation, or writing of the report. The corresponding author had full access to all the data in the study and had final responsibility for the decision to submit for publication.

\section{Results}

We report the clinical course and outcome of the 81 patients who were recruited and have follow-up data. The characteristics of the two study groups (BR and DT) are shown in Table 1. Patients were recruited between 2004 and 2011. As anticipated there were more females than males and a greater proportion were over the age of 10 years at diagnosis. Mean (S.D.) TSH concentrations at the baseline visit were $0.043 \mathrm{mU} / \mathrm{L}(0.052)$ and $0.028 \mathrm{mU} / \mathrm{L}$ (0.024) with some patients taking ATD at the time of this assessment. The recruitment flow diagram for the clinical trial is shown in Fig. 1. Forty-eight patients (23 BR, 25 DT) had thyrotropin receptor antibodies (TRAb) measured during the study period with 44 found to be positive ( 20 BR, 24 DT).

Attendance varied considerably between participants but the median number of scheduled visits during the

Table 1 Patient baseline characteristics of the two groups (BR and DT).

\begin{tabular}{|c|c|c|c|c|}
\hline \multirow[b]{3}{*}{ Variables } & \multicolumn{4}{|c|}{ Treatment, $\boldsymbol{n}(\%)$} \\
\hline & \multicolumn{2}{|c|}{$\mathrm{BR}$} & \multicolumn{2}{|c|}{ DT } \\
\hline & Primary data* & Follow-up** & Primary data* & Follow-up** \\
\hline \multicolumn{5}{|l|}{ Sex } \\
\hline Female & 30 & 31 (77.5) & 30 & $31(75.6)$ \\
\hline Male & 9 & $9(22.5)$ & 8 & $10(24.4)$ \\
\hline \multicolumn{5}{|l|}{ Age, years } \\
\hline$<10$ & 5 & 5 (12.5) & 7 & $7(17.1)$ \\
\hline$>10$ & 34 & $35(87.5)$ & 31 & $34(82.9)$ \\
\hline Mean (S.D.) & $12.9(2.6)$ & & $12.4(3.5)$ & \\
\hline \multicolumn{5}{|c|}{ Free T4 level, pmol/L } \\
\hline$<50$ & 19 & $20(50.0)$ & 20 & $22(53.7)$ \\
\hline$>50$ & 20 & $20(50.0)$ & 18 & $19(46.3)$ \\
\hline Mean (S.D.) & $57.5(27.1)$ & & $57.3(34.8)$ & \\
\hline
\end{tabular}




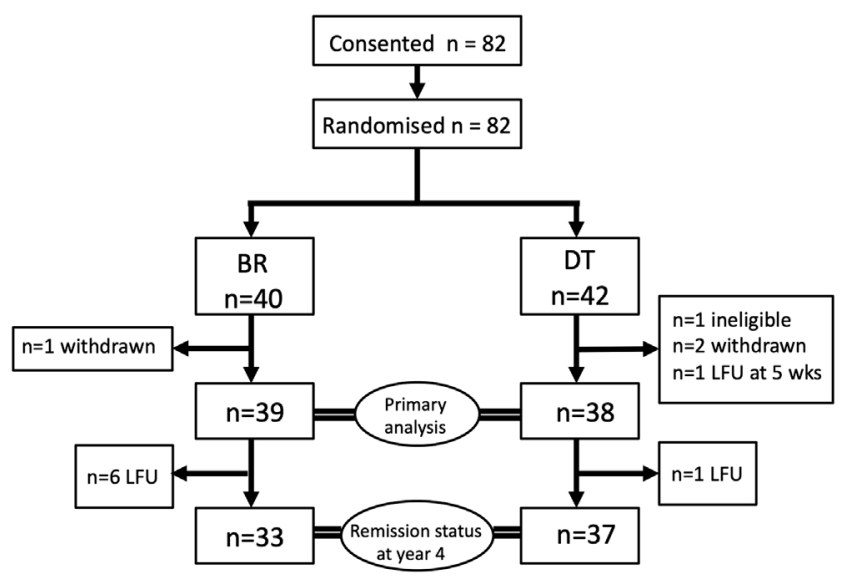

Figure 1

Recruitment flow diagram for the clinical trial.

period of analysis (between 6 months and three years) was the same in the two groups with a median of 10 visits out of the expected 11 in the BR group (range: 3-11) and a median of 10 out of the expected 11 in the DT group (range: 5-11). There were a total of 24 additional unscheduled visits over the same period in the BR group and 16 in DT.

The mean CBZ dose between 6 months and 3 years was $0.61 \mathrm{mg} / \mathrm{kg}$ in the BR group and $0.30 \mathrm{mg} / \mathrm{kg}$ in DT. At the start of the period of biochemical analysis at 6 months, 11 patients in the BR group and 11 in DT still had a suppressed TSH concentration with 5 patients in the BR group yet to commence thyroxine replacement. The mean percentage of TSH measurements within the reference range was $63.8 \%$ in participants allocated to DT compared to $60.2 \%$ on BR. The absolute adjusted difference was $4.3 \%$ in favour of DT (95\% CI: $-7.8 \%$ to $+16.4 \% ; P=0.48$ ) after adjustment for stratification factors. Omitting values obtained following definitivetreatment gave very similar results: mean difference of $4.3 \%$ in favour of DT ( $95 \% \mathrm{CI}:-8.1 \%$ to $+16.6 \% ; P=0.50)$. Figure $2 \mathrm{~A}$ shows the distribution of the proportions of $\mathrm{TSH}$ values on a patient that are within the reference range. Similarly, there was no evidence of a difference in the proportion of FT4 concentrations within the reference range between the two groups, with an adjusted mean difference of $6.8 \%$ in favour of DT $(95 \%$ CI: -2.0 to +15.6 ; $P=0.13$, Fig. 2B). The sensitivity analysis suggested that $11.6 \%$ more FT4 values were within the reference range for those patients on DT $(95 \% \mathrm{CI}$ : $-0.1 \%$ to $+23.4 \%$; $P=0.05)$. The analyses of all four additional measures of control were consistent with the above results, with none providing evidence of a difference between BR and DT.
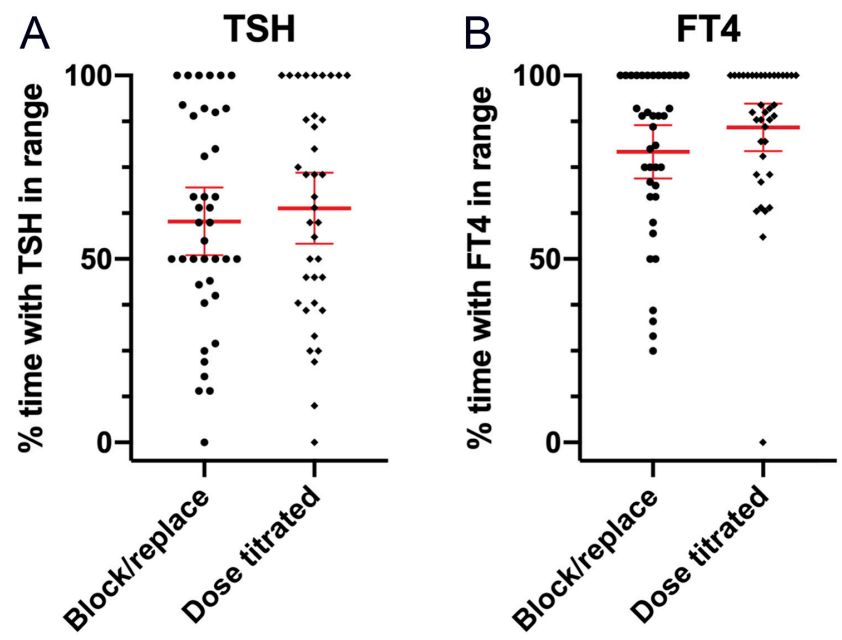

\section{Figure 2}

Depicting the percentage of occasions that serum TSH (A) and FT4 (B) were within the local laboratory reference range in the two study groups (BR and DT). The red, horizontal bars represent mean and $95 \% \mathrm{Cls}$.

Of the 81 patients recruited, 4 withdrew from the trial or were ineligible (three patients withdrew in the first year because of a reluctance to attend study visits and to take medication; one patient was diagnosed with thyroid hormone resistance and was excluded). Of the remaining 77 patients, 6 (15\%) in the BR group and 10 (26\%) in the DT group were known to be in remission at the end of year 4: difference 11\%, 95\% CI (-7\% to 29\%) (Fig. 3) 41 patients had relapsed and 13 patients remained on ATD in breach of the trial protocol because of reluctance to stop treatment or because of issues such as failure of biochemical control on lower ATD doses, concurrent or impending key life events. Seven patients were lost to follow-up prior to

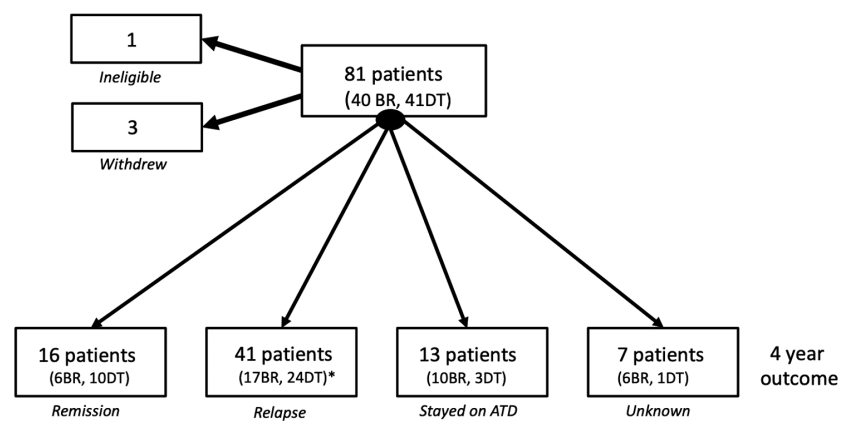

* Of the patients who relapsed 22 returned to ATD (6BR, 16DT), 11 underwent surgery (5 BR, 6DT) and 8 received radio-iodine (6 BR, 2DT)

Figure 3

Four-year outcome in the 81 patients recruited as part of the clinical trial. 
Table 2 Summary of adverse event data.

\begin{tabular}{|c|c|c|}
\hline & \multicolumn{2}{|c|}{ Anti-thyroid drug regimen } \\
\hline & $\overline{B R}$ & DT \\
\hline Non-serious AEs & 200 & 226 \\
\hline AEs related/possibly related to treatment & 78 & 57 \\
\hline Patients experiencing non-serious AEs, $n$ & $34 / 40$ & $36 / 41$ \\
\hline \multicolumn{3}{|l|}{$\begin{array}{l}\text { Patients with expected AEs commonly } \\
\text { linked to thionamide therapy, } n\end{array}$} \\
\hline Rash & 6 & 9 \\
\hline Headache & 8 & 9 \\
\hline Joint related & 5 & 5 \\
\hline Gastrointestinal upset & 5 & 5 \\
\hline Serious AEs & 5 & 3 \\
\hline $\begin{array}{l}\text { Serious AEs related/possibly related } \\
\text { to treatment }\end{array}$ & $\begin{array}{l}3 \text { with neutropenia } \\
(<1000 / \mu \mathrm{L})\end{array}$ & $\begin{array}{l}1 \text { with fever and sore throat, hoarse } \\
\text { voice, mild cough, difficulty swallowing. } \\
1 \text { admitted with excessive vomiting and } \\
\text { then with frontal headache, vomiting, } \\
\text { blurred vision and palpitations }\end{array}$ \\
\hline
\end{tabular}

year 4 but had biochemical data that could be analysed whilst they were treated with ATD. Of the 41 patients who relapsed, 22 (7 BR) returned to ATD therapy, 11 (6 $\mathrm{BR})$ underwent surgery (total thyroidectomy) and 8 (5BR) received radioiodine therapy.

Adverse events in the two trial arms are presented in Table 2. Five patients in the BR group and 2 in DT changed from CBZ to PTU during the trial. There were 426 nonserious AEs recorded from 71 of 81 patients with 200 in the BR group and 226 in the DT group. The number of adverse events deemed to be related or possibly related to treatment with ATD was 78 in the BR group compared to 57 in the DT group. A total of 35 (of 40) in the BR group and 36 (of 41) in the DT group experienced at least one non-serious AE.

There were five serious adverse events (SAEs) from five patients in the BR group with three related or possibly related to trial medication. All 3 SAEs considered to be related to ATD involved the development of neutropenia (neutrophil count less than 1000 per $\mu \mathrm{L}$ ) in the BR group. The three patients were taking 15, 30 and $40 \mathrm{mg}$ of CBZ once daily. One of these patients (on $30 \mathrm{mg}$ CBZ daily) remained on ATD with the neutrophil count recovering spontaneously. There were four SAEs from three patients in the DT group with three considered to be related or possibly related to trial medication. One involved the development of a sore throat but the patient was not neutropenic and classified as unrelated. The remaining two serious AEs (same patient) involved vomiting and an associated admission to hospital and in the second instance vomiting and headache. The development of a rash did not result in permanent discontinuation of ATD in any patient.

\section{Discussion}

Children and adolescents with thyrotoxicosis are often treated with ATD for a lengthy period of time because the remission rate after a 2- to 3-year course of ATD treatment is typically only between 20 and 30\%. The likelihood of remission appears to increase with the duration of therapy $(7,8)$. Knowing how best to administer ATD in young people is therefore important. This trial is the largest prospective study comparing the two ATD regimens (BR and DT) that has ever been conducted in young people and is the first to provide level-1 evidence. The trial has shown no evidence to suggest that there is an important difference in biochemical control in young people with thyrotoxicosis treated with BR when compared to DT. The trial, which was designed to detect a difference between the BR and DT regimens of at least $10 \%$ in the proportion of time in control, has shown that the difference in favour of BR is no greater than $8 \%$ and that in favour of DT could be as large as $16 \%$. In the case of both TSH and FT4 concentrations, the CI indicates that any difference in favour of BR is less than the minimum clinically important difference specified when planning the study. While there could be a difference in favour of BR, its size would not be clinically important, whereas the difference in favour of DT could be much more substantial.

Furthermore, there is no evidence that either adverse events or remission rates are superior in BR patients compared to DT patients. The patients who developed neutropaenia were in the BR group with a larger number of adverse events deemed to be related or possibly related to treatment with ATD in the BR group as well. 
A sensitivity analysis suggested that the proportion of time that FT4 was within the normal range could be higher on DT, with an advantage in favour of BR being very small. This potential outcome was anticipated when designing the trial and was one reason why TSH concentrations were chosen as the primary endpoint. Replacing thyroid hormone when there is no endogenous thyroid hormone release may require relatively high FT4 levels to normalise TSH (12). This is because endogenous thyroid hormone release includes both $\mathrm{T} 4$ and tri-iodothyronine (T3). We do not, therefore, feel that this is evidence in favour of the DT strategy. The two different approaches (BR and DT) have been the subject of a systematic review in adults which led to the recommendation that the DT approach be used because it was associated with fewer side-effects $(13,14)$. This may be because the DT approach is associated with a smaller dose of ATD (15, 16) although clinicians have highlighted the fact that some studies incorporated in the systematic review that formed the basis for the American Thyroid Association (ATA) recommendation administered higher doses of ATD (such as CBZ $100 \mathrm{mg}$ daily) than normally used during routine clinical practice $(17,18)$. The DT approach is also recommended by the European Thyroid Association (19) and American Thyroid Association (ATA) in young people for the same reason (18), although the studies analysed as part of the systematic review were conducted primarily in adults and it has been argued that BR may be associated with improved biochemical stability with the potential for fewer hospital appointments and blood tests as a result $(17,20)$. There are retrospective paediatric (20) and adult (21) studies showing fewer clinic visits in patients managed with a BR regimen although it is unclear to what extent differences were clinically meaningful (21). A recent audit suggested improved biochemical control in paediatric patients managed with BR but patient numbers were small and individuals managed with BR had been on ATD for longer (22). Paediatric endocrinologists in the UK still use the BR strategy (23) which may indicate that some clinicians feel that BR is a useful strategy in selected patients where DT does not result in biochemical stability.

The trial that we report here provides the first level 1 evidence to support the recommendation of the ATA guideline to use a DT regimen on the basis that the risk of adverse events is greater with BR. In the present study, three patients in the BR arm developed neutropenia in comparison to none in the DT group although the overall number of AEs was similar in the two groups.

Childhood Graves' disease has a worse outlook than the equivalent condition in older people, with several studies showing a remission rate of $25 \%$ or less after 2 years' ATD therapy $(7,8,24)$ compared to figures around $50 \%$ in adults. The reasons for this are unclear but it could reflect the fact that younger people tend to have more severe thyrotoxicosis at presentation than adults, that the recognition is frequently delayed or that the compliance with disease-modifying ATD medication may be suboptimal, particularly during teenage years. There was no evidence of a difference in the proportion of patients in remission in the two groups that we studied: but CIs are large such that the remission rate could be up to $7 \%$ greater on BR or up to $29 \%$ greater on DT. This is in keeping with earlier data from adult studies (14). It is interesting to note that the proportion in remission (20\%) is of a similar order of magnitude to those reported in the paediatric literature.

We found that young people with thyrotoxicosis are a challenging group of patients to study. Therapeutic changes may be delayed by school examinations, moving into higher education or travelling, and many appeared to struggle to attend out-patient appointments as scheduled. Monitoring patient progress for a period of 4 years was not always straight-forward in these young people. We suspect that the fact that 13 patients opted to stay on ATD at the end of the initial 3-year period was linked to the desire to maintain biochemical stability at an important stage of the young person's life. This trial has a number of weaknesses that we have alluded to above. First, whilst the trial did not recruit as many subjects as hoped this is still the largest ever randomised trial of the two key treatment approaches conducted in young people. Secondly, whilst patients were diagnosed with thyrotoxicosis, not all had thyroid receptor antibodies measured and so it is possible that some young people had self-limiting thyroiditis (25). Not all laboratories could access a thyroid receptor antibody assay when the trial commenced but it is of note that patients were recruited by paediatric endocrinologists who will be familiar with the spectrum of thyroid disease in the young and when TRAb were measured they were raised or positive in $92 \%$ of participants. Finally, thyrotoxicosis in young people due to Graves' Disease is characterised by elevated serum FT3 concentrations and we would ideally have measured FT3 levels and used this as one of the secondary outcome measures. When the trial commenced many laboratories did not measure FT3 concentrations.

This study has not shown any evidence to refute the current guidelines that recommend DT in most growing people with thyrotoxicosis. There is no evidence from this trial to suggest that BR is associated with important improvements in biochemical stability compared with DT. 


\section{Supplementary materials}

This is linked to the online version of the paper at https://doi.org/10.1530/ EJE-20-0617.

\section{Declaration of interest}

T C and S P were members of the NICE Thyroid guideline committee 2019. No competing financial interests exist.

\section{Funding}

This trial was funded by both the British Thyroid Foundation and by the Child Growth Foundation. C W was funded by the Medical Research Council/MDUK (MR/N020588/1).

\section{Acknowledgements}

The authors would like thank the patients who participated in the trial. The authors would also like to acknowledge the input of members of the BSPED study team including Amelia Mayo, Craig Oxley (Aberdeen), Jeremy Kirk, Tim Barrett (Birmingham), Carlo Acerini (Cambridge); John Gregory, Justin Warner (Cardiff); Heather Stirling (Coventry); Stephen Greene (Dundee); Amanda Drake (Edinburgh); Guftar Shaikh, Wendy Paterson, Emma-Jane Gault (Glasgow); Jon Staines (Kilmarnock); Joanne Blair and Poonam Dharmaraj (Liverpool); Indi Banerjee, Sarah Ehtisham (Manchester); Helen Johnstone, Debbie Matthews, Kate Owen (Newcastle); Nandu Thalange (Norwich); Paul Dimitri, Neil Wright, Jerry Wales (Sheffield); Assunta Albanese, Murray Bain (St. Georges) as well as the specialist nurse teams who were involved in the process of supporting patients and collecting data. The authors would also like to acknowledge the support of Cambridge and Newcastle clinical trials units as well as Stephen Bruce, Neil Davidson, Julie Edge, Eve Smith and the members of the steering and data monitoring committees.

\section{References}

1 Rivkees SA \& Mattison DR. Ending propylthiouracil-induced liver failure in children. New England Journal of Medicine $2009 \mathbf{3 6 0}$ 1574-1575. (https://doi.org/10.1056/NEJMc0809750)

2 Schenk D, Donaldson M \& Cheetham T. Which antithyroid drug regimen in paediatric Graves' disease? Clinical Endocrinology 201277 806-807. (https://doi.org/10.1111/j.1365-2265.2012.04509.x)

3 Sohal APS, Dasarathi M, Lodh R, Cheetham T \& Devlin AM. Speech and language delay in two children: an unusual presentation of hyperthyroidism. Journal of Pediatric Endocrinology and Metabolism 201326 1171-1174. (https://doi.org/10.1515/jpem-2013-0050)

4 Lillevang-Johansen M, Abrahamsen B, Jørgensen HL, Brix TH \& Hegedüs L. Duration of hyperthyroidism and lack of sufficient treatment are associated with increased cardiovascular risk. Thyroid 201929 332-340. (https://doi.org/10.1089/thy.2018.0320)

5 Williams GR \& Bassett JHD. Thyroid diseases and bone health. Journal of Endocrinological Investigation 201841 99-109. (https://doi. org/10.1007/s40618-017-0753-4)

6 Okosieme OE, Taylor PN, Evans C, Thayer D, Chai A, Khan I, Draman MS, Tennant B, Geen J, Sayers A et al. Primary therapy of Graves' disease and cardiovascular morbidity and mortality: a linked-record cohort study. Lancet: Diabetes and Endocrinology 20197 278-287. (https://doi.org/10.1016/S2213-8587(19)30059-2)

7 Léger J, Gelwane G, Kaguelidou F, Benmerad M, Alberti C \& French Childhood Graves' Disease Study Group. Positive impact of long-term antithyroid drug treatment on the outcome of children with Graves' disease: national long-term cohort study. Journal of
Clinical Endocrinology and Metabolism 201297 110-119. (https://doi. org/10.1210/jc.2011-1944)

8 Léger J \& Carel JC. MANAGEMENT OF ENDOCRINE DISEASE: Arguments for the prolonged use of antithyroid drugs in children with Graves' disease. European Journal of Endocrinology 2017177 R59-R67. (https://doi.org/10.1530/EJE-16-0938)

9 Lazar L, Kalter-Leibovici O, Pertzelan A, Weintrob N, Josefsberg Z \& Phillip M. Thyrotoxicosis in prepubertal children compared with pubertal and postpubertal patients. Journal of Clinical Endocrinology and Metabolism 200085 3678-3682. (https://doi.org/10.1210/ jcem.85.10.6922)

10 Evans S, Royston P \& Day S. Minim: allocation by minimisation in clinical trials, 2019.

11 StataCorp. Stata Statistical Software: Release 14. STATAcorp LP, 2015.

12 Fish LH, Schwartz HL, Cavanaugh J, Steffes MW, Bantle JP \& Oppenheimer JH. Replacement dose, metabolism, and bioavailability of levothyroxine in the treatment of hypothyroidism. Role of triiodothyronine in pituitary feedback in humans. New England Journal of Medicine 1987316 764-770. (https://doi.org/10.1056/ NEJM198703263161302)

13 Abraham P, Avenell A, Park CM, Watson WA \& Bevan JS. A systematic review of drug therapy for Graves' hyperthyroidism. European Journal of Endocrinology 2005153 489-498. (https://doi.org/10.1530/ eje.1.01993)

14 Abraham P, Avenell A, McGeoch SC, Clark LF \& Bevan JS. Antithyroid drug regimen for treating Graves' hyperthyroidism. Cochrane Database of Systematic Reviews 2010 CD003420. (https://doi. org/10.1002/14651858.CD003420.pub4)

15 Cooper DS, Goldminz D, Levin AA, Ladenson PW, Daniels GH, Molitch ME \& Ridgway EC. Agranulocytosis associated with antithyroid drugs. Effects of patient age and drug dose. Annals of Internal Medicine 198398 26-29. (https://doi.org/10.7326/0003-481998-1-26)

16 Takata K, Kubota S, Fukata S, Kudo T, Nishihara E, Ito M, Amino N $\&$ Miyauchi A. Methimazole-induced agranulocytosis in patients with Graves' disease is more frequent with an initial dose of $30 \mathrm{mg}$ daily than with $15 \mathrm{mg}$ daily. Thyroid 200919 559-563. (https://doi. org/10.1089/thy.2008.0364)

17 Razvi S, Vaidya B, Perros P \& Pearce SHS. What is the evidence behind the evidence-base? The premature death of block-replace antithyroid drug regimens for Graves' disease. European Journal of Endocrinology 2006154 783-786. (https://doi.org/10.1530/eje.1.02169)

18 Ross DS, Burch HB, Cooper DS, Greenlee MC, Laurberg P, Maia AL, Rivkees SA, Samuels M, Sosa JA, Stan MN et al. 2016 American Thyroid Association guidelines for diagnosis and management of hyperthyroidism and other causes of thyrotoxicosis. Thyroid 201626 1343-1421. (https://doi.org/10.1089/thy.2016.0229)

19 Kahaly GJ, Bartalena L, Hegedüs L, Leenhardt L, Poppe K \& Pearce SH. 2018 European Thyroid Association guideline for the management of graves' hyperthyroidism. European Thyroid Journal 20187 167-186. (https://doi.org/10.1159/000490384)

20 Raza J, Hindmarsh PC \& Brook CGD. Thyrotoxicosis in children: thirty years' experience. Acta Paediatrica 199988 937-941. ( https:// doi.org/10.1111/j.1651-2227.1999.tb00185.x)

21 Vaidya B, Wright A, Shuttleworth J, Donohoe M, Warren R, Brooke A, Gericke CA \& Ukoumunne OC. Block and replace regime versus titration regime of antithyroid drugs for the treatment of Graves' disease: a retrospective observational study. Clinical Endocrinology 201481 610-613. (https://doi.org/10.1111/cen.12478)

22 Vigone MC, Peroni E, Frenna M Di, Mora S, Barera G \& Weber G. 'Block-and-replace' treatment in Graves' disease: experience in a cohort of pediatric patients. Journal of Endocrinological Investigation 202043 595-600. (doi:10.1007/s40618-019-01144-0)

23 Lawrence N, Cheetham T \& Elder C. How do paediatricians use and monitor antithyroid drugs in the UK? A clinician survey. 
Clinical Endocrinology 201991 417-423. (https://doi.org/10.1111/ cen.14046)

24 Ohye H, Minagawa A, Noh JY, Mukasa K, Kunii Y, Watanabe N, Matsumoto M, Suzuki M, Yoshihara A, Ito K et al. Antithyroid drug treatment for Graves' disease in children: a long-term retrospective study at a single institution. Thyroid 201424 200-207. (https://doi. $\operatorname{org} / 10.1089 /$ thy.2012.0612)
25 Kourime M, McGowan S, Towati MAl, Ahmed SF, Stewart G, Williamson S, Hunter I \& Donaldson MDC. Long-term outcome of thyrotoxicosis in childhood and adolescence in the west of Scotland: the case for long-term antithyroid treatment and the importance of initial counselling. Archives of Disease in Childhood 2018103 637-642. (https://doi.org/10.1136/ archdischild-2017-313454)

Received 3 June 2020

Revised version received 3 September 2020

Accepted 18 September 2020 